\title{
Nonlinear Magnetoelectric Response Characteristics of Galfenol-piezoelectric Composite Sensors
}

\author{
Zhiwen Zhu, ${ }^{1}$ Yaqin Wen, ${ }^{1}$ Hui Sheng, ${ }^{1}$ and Jia $\mathrm{Xu}^{2 *}$ \\ ${ }^{1}$ Department of Mechanics, Tianjin University, \\ 92 Weijin Road, Nankai District, Tianjin 300072, China \\ ${ }^{2}$ Tianjin Key Laboratory of Nonlinear Dynamics and Control, Tianjin University, \\ 92 Weijin Road, Nankai District, Tianjin 300072, China
}

(Received April 5, 2017; accepted March 5, 2018)

Keywords: Galfenol, piezoelectric ceramics, hysteretic nonlinearity, magnetoelectric response

A kind of Galfenol-piezoelectric composite sensor is proposed, and its nonlinear magnetoelectric response characteristics are analyzed in this paper. New nonlinear differential terms with fractional index are proposed to explain the hysteresis of Galfenol's strain-magnetic field intensity (MFI) curves and the piezoelectric zirconate titanate (PZT)'s displacementvoltage curves, and a nonlinear dynamic model for such a sensor under a harmonic magnetic field is developed when the electromagnetic interference (EMI) is considered. The expression of the system's dynamic response is obtained, and its bifurcation is discussed. Finally, the simulation results are proved by experiments. The theoretical and experimental results show that the intensity of EMI plays an important role in the system's response. These results may be helpful for the optimal design and improvement of Galfenol-piezoelectric composite sensors.

\section{Introduction}

A highly sensitive magnetic sensor is an important component in modern electronic products. It is widely used to measure magnetic field intensity (MFI) to determine current, location, direction, and other physical parameters. The traditional magnetic sensor is a Hall component, which has good accuracy and a simple structure. However, the Hall component requires an external power supply, and is easily affected by temperature. The giant magnetostrictive-piezoelectric composite sensor is a new kind of magnetic sensor. It has high accuracy and self-generating characteristics, and is used widely where the power supply is very difficult to apply. The first kind of giant magnetostrictive material applied in sensors is Terfenol-D (Tb-Dy-Fe alloy). Although Terfenol-D has high compressive strength (700 MPa), its tensile strength is only $28 \mathrm{Mpa}$. It means that Terfenol-D cannot withstand tension load, which limits its application in engineering fields. Galfenol is a novel giant magnetostrictive material. It has both high compressive strength (700 MPa) and high tensile strength (500 MPa), which causes it be a promising material for sensors and actuators.

*Corresponding author: e-mail: xujia@tju.edu.cn https://dx.doi.org/10.18494/SAM.2018.1902 
Many researchers have studied the characteristics of magnetostrictive-piezoelectric sensors. Prieto et al. reduced the hysteresis in magnetostrictive-piezoelectric magnetic sensors, ${ }^{(1)}$ and improved their magnetic coefficients by removing the viscous interface. ${ }^{(2)}$ Ueno and Higuchi proposed a kind of highly sensitive and heat-resistant magnetic sensor using a magnetostrictivepiezoelectric laminate composite. ${ }^{(3)}$ Huang et al. designed a new class of passive magnetic field sensors based on combinations of magnetostrictive and electroactive materials. ${ }^{(4)}$ In recent years, Zhang et al. have improved the magnetoelectric effects in magnetostrictive-piezoelectric composites combined with flux concentration effects. ${ }^{(5)}$ Elhosni et al. established a finite element method (FEM) model of magnetic field surface acoustic wave (SAW) sensors based on magnetostrictive-piezoelectric layered structures, and validated the model by experiments. ${ }^{(6)}$ $\mathrm{Xu}$ et al. discussed the nonlinear response characteristics of giant magnetostrictive-piezoelectric composite sensors. ${ }^{(7)}$ Although many achievements in magnetostrictive-piezoelectric composite sensors have been reported, ${ }^{(8-15)}$ theoretical results on the dynamic characteristics of the magnetostrictive-piezoelectric composite sensors subjected to electromagnetic interference (EMI) are few.

There is always EMI in engineering fields, which can be regarded as a random perturbation. In this paper, a new Galfenol-piezoelectric composite sensor is proposed, and its dynamic responses under a harmonic magnetic field are analyzed when the EMI is considered. A new differential term is introduced to describe the hysteretic phenomena of Galfenol's strain-MFI curves. The stability of the system is analyzed, and the effects of the EMI intensity on the system's response are discussed.

\section{Nonlinear Dynamic Model of Galfenol-piezoelectric Composite Sensor}

The structure of a Galfenol-piezoelectric composite sensor is shown in Fig. 1. When the sensor is placed into a changing magnetic field, the Galfenol rod moves, and then the piezoelectric zircouate titanate (PZT) stack is subjected to changing pressure, which produces changing currents. The Galfenol's strain-MFI curves are shown in Fig. 2. The curves clearly have hysteretic characteristics.

In 1998, Jin et al. proposed a new nonlinear viscoelasticity model as follows to describe the hysteresis phenomena of Hertzian contact force $F(x)$ in the collision process. ${ }^{(16)}$

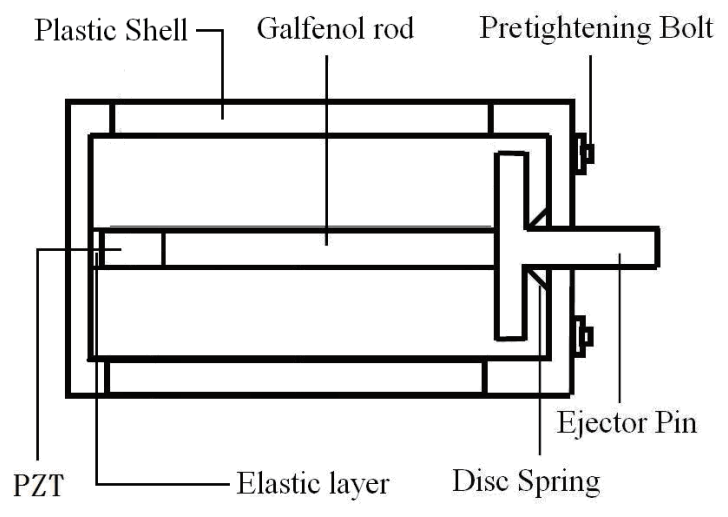

Fig. 1. Structure of Galfenol-piezoelectric composite sensor.

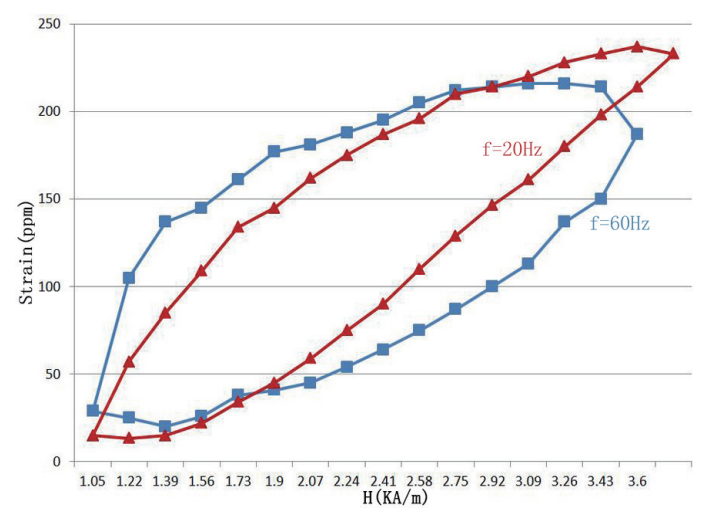

Fig. 2. (Color online) Galfenol's strain-MFI curves. 


$$
F(x)=k_{0} x^{\frac{3}{2}}+c_{v} x^{\frac{3}{2}} \dot{x}
$$

Here, we use the above model to describe Galfenol's strain-MFI curves as follows:

$$
\varepsilon=a_{1} H^{\frac{3}{2}}+a_{2} H^{\frac{3}{2}} \dot{H}
$$

where $\varepsilon$ is the strain, $H$ is the MFI, and $a_{i}(i=1,2)$ are the coefficients determined by the hysteretic loop.

In addition, the displacement-voltage characteristics of PZT ceramics also cannot be regarded as linear. In fact, even the polarized PZT ceramics also has slight hysteretic characteristics. The displacement-voltage characteristics of PZT ceramics stacking are shown in Fig. 3.

Similarly, we can establish the displacement-voltage model of PZT as follows:

$$
U=b_{1} x^{\frac{3}{2}}+b_{2} x^{\frac{3}{2}} \dot{x}
$$

where $U$ is the output voltage of PZT stacking, $x$ is the displacement of PZT stacking, and $b_{i}(i=1,2)$ are the coefficients determined by the hysteretic loop.

Now, we can establish the sensor's dynamic model. In this study, the Hamilton variational principle is applied to system modeling as follows:

$$
\delta S=\int \delta\left(T_{1}+T_{2}+T_{3}+M^{K}-U_{1}-U_{2}-U_{3}-M^{P}-W_{d}+W_{O E}\right) d t=0
$$

where $T_{i}$ is the kinetic energy of the three materials, $T_{i}=\frac{1}{2} \int_{0}^{L_{i}} \rho_{i} A_{i}\left(\frac{d x_{i}}{d t}\right)^{2} d l_{i},(i=1,2,3), 1: \mathrm{PZT}$ stacking; 2: elastic layer; 3: Galfenol rod. $L_{i}$ is the length of the three materials, $\rho_{i}$ is the density, $A_{i}$ is the area, and $x_{i}$ is the displacement. $M^{K}$ is the magnetic field energy of the Galfenol rod,

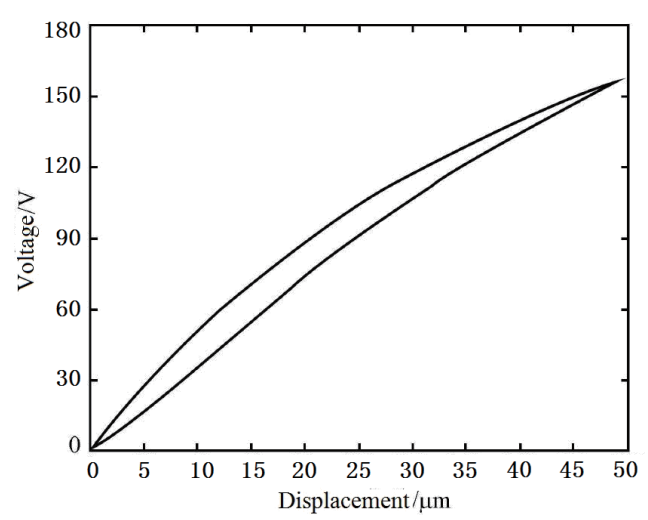

Fig. 3. Displacement-voltage curves of PZT. 
$M^{K}=\frac{1}{2} \iiint B \cdot K d V, K=K_{0} \sin \Omega t$ is the intensity of the magnetic field; $U_{i}$ is the potential energy of the three materials, $U_{i}=\frac{1}{2} \int_{0}^{L_{i}} \frac{E_{i} A_{i}}{l_{i}} x_{i}^{2} d l_{i},(i=1,2,3), E_{i}$ is the elastic modulus; $M^{P}$ is the electric field energy of PZT, $M^{P}=\frac{1}{2} \iiint E_{3} \cdot D d V=\frac{A_{1}}{2} \int_{0}^{L_{1}} E_{33} D_{33} d l_{1}, E_{33}=\frac{U}{L_{1}}$ is the intensity of the electric field, $U$ is the output voltage of PZT stacking, $D_{33}$ is the electric

displacement. $W_{d}$ is the work done by the system's damp force, $W_{d}=\int_{0}^{L_{1}+L_{2}+L_{3}} c q \frac{\partial q}{\partial t} d l$, $q=x_{1}+x_{2}+x_{3}, c$ is the damping coefficient of the structures; $W_{O E}$ is the work done by the outside excitation, $W_{O E}=\int_{0}^{L} F u d x, F$ is the stochastic perturbation caused by EMI, $F=\bar{F} \varsigma(t)$, where $\varsigma(t)$ is the normal Gauss white noise.

Let the displacement of the rod end be the system's response $q$. We can obtain the dynamic equation of the system's response as

$$
\ddot{q}+\left(2 \eta+c_{2} q^{\frac{3}{2}}\right) \dot{q}+c_{1} q+c_{3} q^{\frac{3}{2}}=\bar{K} \sin \Omega t+e \varsigma(t),
$$

where $c_{1}=\frac{\sum_{i=1}^{3} E_{i} A_{i}}{\left(\sum_{i=1}^{3} \rho_{i} L_{i} A_{i}\right)^{2}}, c_{2}=\frac{\pi b_{0}}{\sum_{i=1}^{3} \rho_{i} L_{i} A_{i}}, c_{3}=\frac{\pi E_{1} a_{0}}{A_{1} \sum_{i=1}^{3} \rho_{i} L_{i} A_{i}}, \bar{K}=\frac{\delta_{G} K E_{3} A_{3}}{\sum_{i=1}^{3} \rho_{i} L_{i} A_{i}}$, and $e=\frac{\pi^{2} \bar{F}}{\sum_{i=1}^{3} \rho_{i} L_{i} A_{i}}$

\section{Dynamic Characteristics of the System}

We can obtain the drift and diffusion coefficients of the Ito stochastic process using the stochastic average method as follows.

$$
\begin{gathered}
m(H)=\left(\frac{D e^{2}}{c_{1}}-2 \eta\right) H-\frac{c_{2}}{\pi}\left(\frac{2}{c_{1}}\right)^{\frac{3}{4}} H^{\frac{7}{4}} \\
\sigma^{2}(H)=\frac{D e^{2}}{c_{1}} H^{2}
\end{gathered}
$$

The averaged Fokker-Planck-Kolmogorov (FPK) equation of Eq. (5) is

$$
\frac{\partial f}{\partial t}=-\frac{\partial}{\partial H}[m(H) f]+\frac{1}{2} \frac{\partial^{2}\left[\sigma^{2}(H) f\right]}{\partial H^{2}},
$$


where $f$ is the stationary probability density (SPD) of the system's response. Thus,

$$
f(H)=\bar{A} H^{-\frac{4 \eta c_{1}}{D e^{2}}} \exp \left[\frac{8 K c_{1} c_{2}}{3 \pi D e^{2}}\left(\frac{2}{c_{1}}\right)^{\frac{3}{4}} H^{\frac{3}{4}}\right] .
$$

The numerical results of the system response are presented in Fig. 4, where $c_{1}=1000, c_{2}=0.05$, $c_{3}=0.3, D=0.5, \eta=0.01$, and $K=0.5$. According to Eq. (9) and Fig. 4, we find that

1) $p=0$ and $q=0$ when $H=0$, given that $H=\frac{1}{2} p^{2}+\frac{1}{2} c_{1} q^{2}$. Thus, the trivial solution $H$ $=0$ corresponds to the origin point $(0,0)$ in the SPD map.

2) $\eta$ is the linear damping coefficient, $\eta \geq 0 ; c_{1}$ is the system's linear stiffness, $c_{1} \geq 0$; and $D$ is the intensity of normal Gauss white noise, $D>0$.

3) There is a loop in the SPD map when $K=0.5$ and $e=0$, which is shown in Fig. 4(a). It means that the system's motion and the output voltage are both periodic when the composite sensor is in a harmonic magnetic field and there is no EMI.

4) There is a crest and a loop in the SPD map when $K=0.5$ and $e=0.2$, which are shown in Fig. 4(b). It means that the system's motion has two possibilities, one is a periodic motion with large amplitude, and the other is vibration near the balance point $(0,0)$. The system's motion is determined by the initial conditions.

5) In the variation process of the system's parameters, the system's response can jump from balance point to periodic orbits under the outside excitation, which causes the

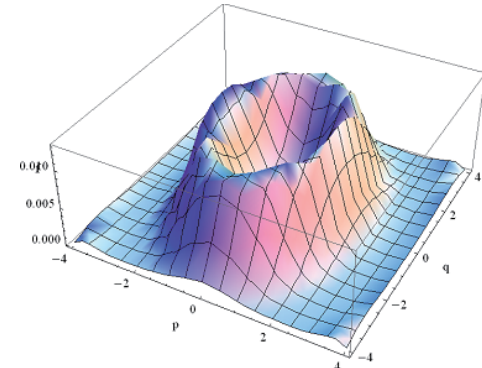

(a)

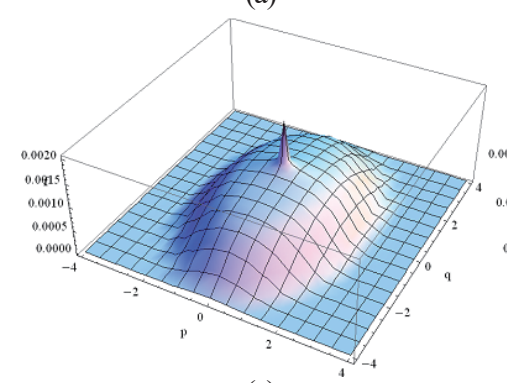

(c)

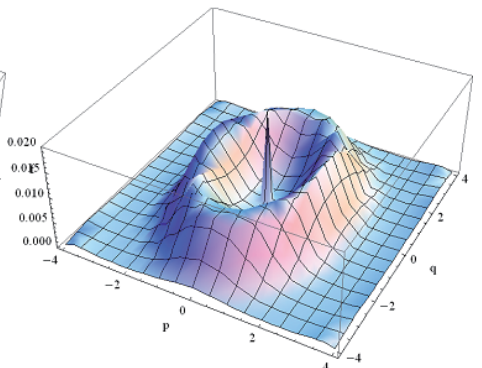

(b)

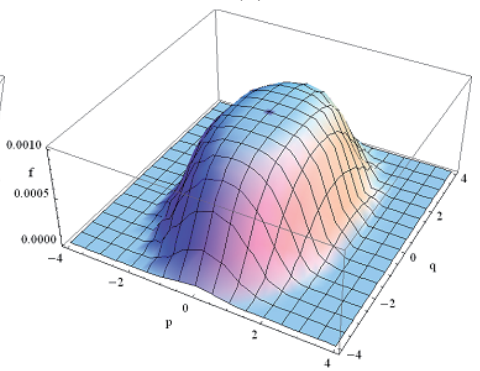

(d)

Fig. 4. (Color online) Probability density of the system's response when $K=0.5$ and (a) $e=0$, (b) $e=0.2$, (c) $e=0.5$, and (d) $e=1$. 
bifurcation of the system.

6) There are a pick and a platform in the SPD map when $K=0.5$ and $e=0.5$, which are shown in Fig. 4(c). The probability of the pick is higher than that of the platform, which means that the system should vibrate slightly near the balance point $(0,0)$.

7) There is a platform in the SPD map when $K=0.5$ and $e=1$, which is shown in Fig. 4(d). Each point in the platform has a high probability. That is, the system's response can appear in any point in the area, which means that the system's motion is random.

The experimental results for a Galfenol-piezoelectric composite sensor subjected to harmonic and random magnetic excitations are shown in Fig. 5. We also note that the system's response becomes random when the noise's intensity further increases.

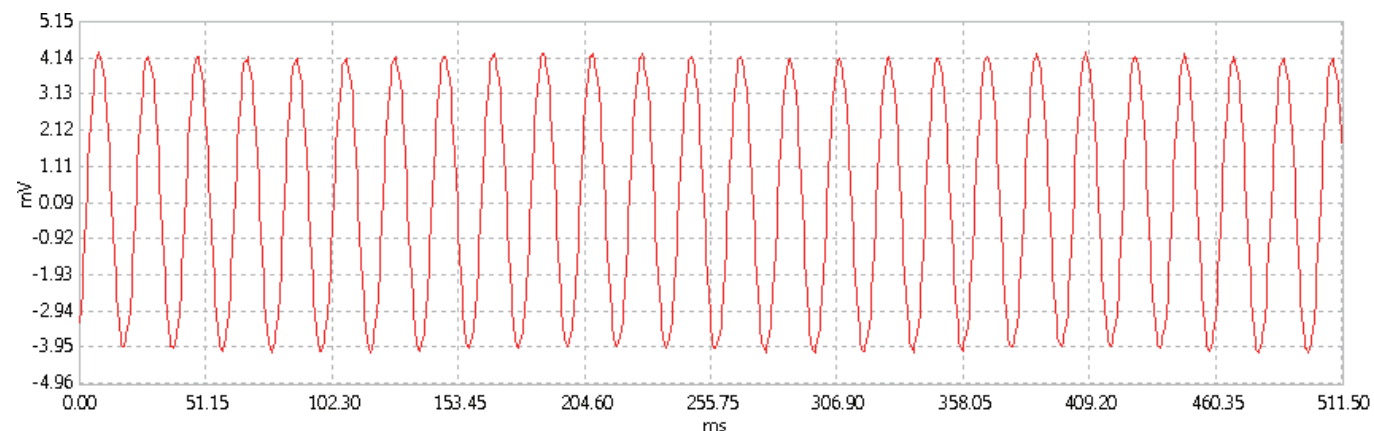

(a)

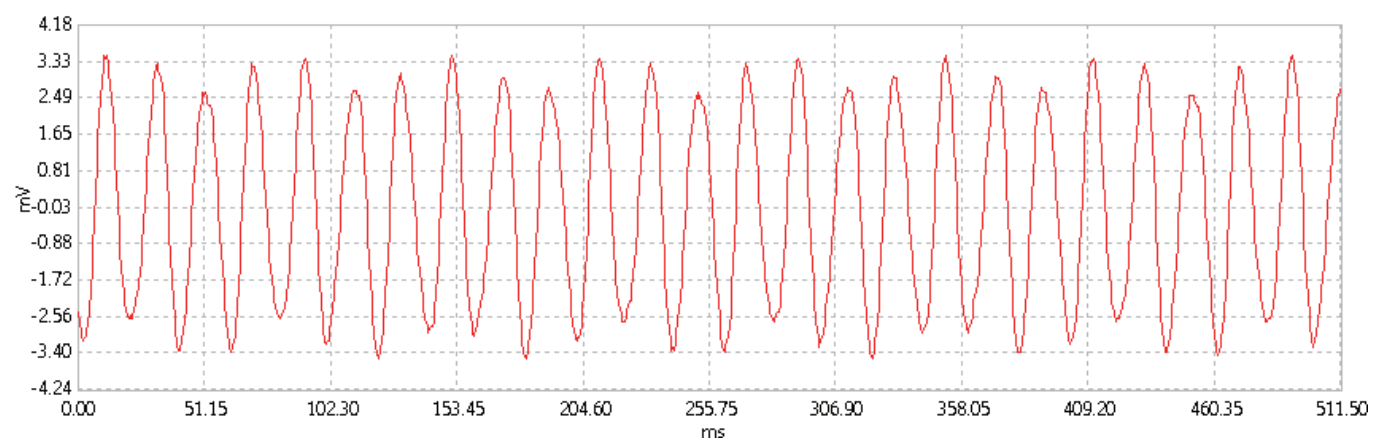

(b)

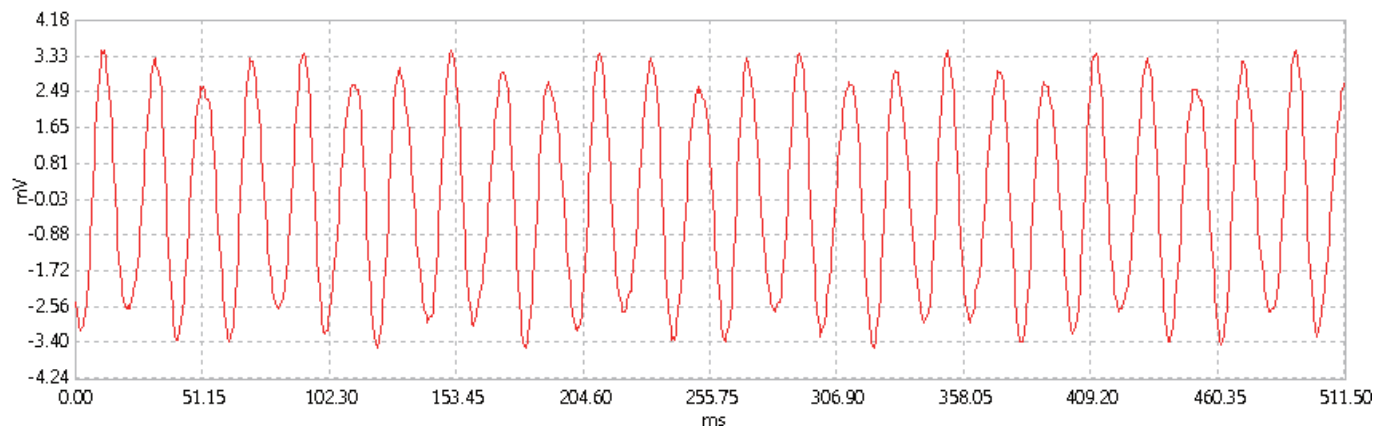

(c)

Fig. 5. (Color online) Output voltage of the composite sensor when $K=0.5$ and (a) $e=0$, (b) $e=0.2$, and (c) $e=0.5$. 


\section{Conclusions}

A kind of Galfenol-piezoelectric composite sensor is proposed, and its nonlinear magnetoelectric response characteristics are analyzed in this paper. New nonlinear differential terms with fractional index are proposed to explain the hysteresis of Galfenol's strain-MFI curves and PZT's displacement-voltage curves, and a nonlinear dynamic model for such a sensor under a harmonic magnetic field is developed when the EMI is considered. The expression of the system's dynamic response is obtained, and its bifurcation is discussed. Finally, the simulation results are proved by experiments. The theoretical and experimental results show that the intensity of EMI plays an important role in the system's response: when the intensity of EMI is sufficiently small, the system's motion is periodic, and the sensor can work properly; when the intensity of EMI is sufficiently large, the system's motion becomes random, and the system's stability is destroyed.

\section{Acknowledgments}

The authors gratefully acknowledge the support of the Natural Science Foundation of China (NSFC) through Grant Nos. 11272229 and 11302144, Chinese Aviation Science Foundation through Grant No. 2016ZA48001, and the Tianjin Research Program of Application Foundation and Advanced Technology through Grant No. 16JCYBJC18800.

\section{References}

1 J. L. Prieto, C. Aroca, and E. Lopez: IEEE Trans. Magn. 34 (1998) 3913.

2 J. L. Prieto, P. Sanchez, and C. Aroca: Sens. Actuators, A 84 (2000) 338.

3 T. Ueno and T. Higuchi: IEEE Trans. Magn. 41 (2005) 3670.

4 J. K. Huang, D. Bono, and R. C. O'Handley: Sens. Lett. Magn. 5 (2007) 105.

5 H. Zhang, C. J. Lu, and C. B. Xu: AIP Adv. 5 (2015) 047114.

6 M. Elhosni, O. Elmazria, and S. Petit-Watelot: Sens. Actuators, A 240 (2016) 41.

7 J. Xu, R. Z. Li, and Z. W. Zhu: Sens. Mater. 27 (2015) 633.

8 D. A. Fillippov: Phys. Solid State 47 (2005) 1080.

9 P. Li, Y. M. Wen, and L. X. Bian: Appl. Phys. Lett. 90 (2007) 022503.

10 V. M. Petrov, G. Srinivasan, and M. I. Bichurin: Phys. Rev. B 75 (2007) 224407.

11 C. S. Lee, J. Joo, and S. Han: Appl. Phys. Lett. 85 (2004) 1841.

12 Z. Y. Jia, W. Liu, and Y. S. Zhang: Sens. Actuators, A 128 (2006) 158.

13 J. Xu, Y. X. Kong, and H. L. Wang: Sens. Mater. 28 (2016) 631.

14 S. Masuda, Y. Matsumura, and Y. Nishi: J. Jpn. Inst. Met. 70 (2006) 166.

15 N. Tiercelin, V. Preobrazhensky, and P. Pernod: J. Magn. Magn. Mater. 210 (2000) 302.

16 D. P. Jin, H. Y. Hu, and Z. Q. Wu: J. Vib. Eng. 11 (1998) 46. 


\section{About the Authors}

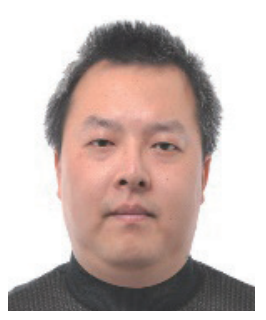

Zhi-Wen Zhu received his B.S. degree from Shanghai Jiaotong University, China, in 1996 and his M.S. and Ph.D. degrees from Tianjin University, China, in 2000 and 2003, respectively. From 2003 to 2005, he was a postdoctoral fellow at Tianjin University, China. From 2005, he was an assistant professor at Tianjin University, and since 2010, he has been an associate professor at Tianjin University. His research interests are in nonlinear dynamics and control of smart structures.

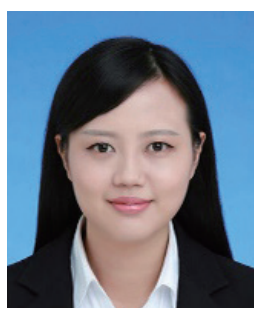

Ya-Qin Wen received her Bachelor's degree in Engineering Mechanics from Xiangtan University, China, in 2016. She is now a postgraduate student in Tianjin University. Her current research interests are in nonlinear dynamics and bifurcation of smart structures.

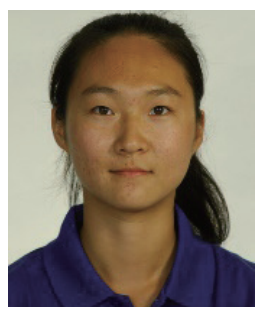

Hui Sheng is an undergraduate student in Tianjin University. Her current research interests are in the nonlinear dynamics responses of smart structures.

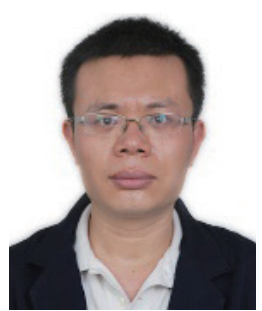

Jia Xu received his B.S., M.S., and Ph.D. degrees from Tianjin University, China, in 2003, 2005, and 2008, respectively. Since 2008, he has been an assistant professor at Tianjin University. His research interests are in stochastic dynamics analysis and control of nonlinear systems. 